\title{
Transition to turbulence in shear flows
}

\author{
Bruno Eckhardt \\ Fachbereich Physik, Philipps-Universität Marburg, 35032 Marburg, Germany
}

\begin{abstract}
Pipe flow and many other shear flows show a transition to turbulence at flow rates for which the laminar profile is stable against infinitesimal perturbations. In this brief review the recent progress in the understanding of this transition will be summarized, with a focus on the linear and nonlinear states that drive the transitions, the extended and localized patterns that appear, and on the spatio-temporal dynamics and their relation to directed percolation.
\end{abstract}

\section{INTRODUCTION}

The ways in which flows become turbulent can broadly be divided into two groups. One group contains all flows where the laminar profile shows a linear instability as the flow rate increases. A subsequent cascade of instabilities results in ever more complicated dynamics, which in spirit, though not in detail, reflects Landau's descriptions of the transition to turbulence [1]. Fluids sheared between independently rotating concentric cylinders (Taylor-Couette flow) or heated from below (Rayleigh-Benard flow) follow this route and the linear and nonlinear properties of the patterns that form have been explored in considerable detail [2]

The second group has pressure driven flow in a pipe as a paradigmatic example [7, 8], but also includes plane Couette flow or boundary layers and several other cases shown in Fig. 1. They all share the feature that turbulence appears for parameters where the laminar profile is still stable. Their transition typically requires finite amplitude perturbations, it shows a sensitive dependence on initial conditions, and there are no simple patterns above the onset [9]. Moreover, the flows show a remarkable variety of spatio-temporal dynamics near onset [10 14].

In the last two decades, various elements for an explanation of the transition in these flows have identified and explored, so that we now have a framework in which pipe flow and other flows can be approached. The following sections provide a brief survey of key concepts such as exact coherent states (ECS), secondary bifurcations and the formation of turbulent transients and spatio-temporal patterns, and the connection to phase transitions of the directed percolation type. Various other aspects of the transition are discussed in [15]24].

In the following sections, we describe the non-normal amplification in the linearized dynamics (section 2), finite amplitude subcritical bifurcations that give rise to exact coherent structures around which turbulence can form (section 3 ), the presence of localized exact coherent structures (section 4), the long-time dynamics of localized structures and their relation to directed percolation (section 5). A few concluding remarks and an outlook on open issues are given in section 6 .

\section{LINEAR APPROACHES}

The linearization of the Navier-Stokes equation around a background shear $\mathbf{U}_{0}$ reads

$$
\partial_{t} \mathbf{u}+\left(\mathbf{U}_{0} \cdot \nabla\right) \mathbf{u}+(\mathbf{u} \cdot \nabla) \mathbf{U}_{0}+\nabla p=\nu \Delta \mathbf{u}
$$

Asymptotic stability requires that all perturbations decay eventually. This does not imply that perturbations decay monotonically: they can transiently be amplified beyond their initial amplitude before they disappear eventually. Typically, the structures that are most efficient in extracting energy from the background shear are downstream vortices which then drive the formation of modulations in the downstream velocity, so-called streaks. This interaction provides the building blocks for the nonlinear states as indicated in Fig. 2.

The significance of vortices and streaks can be studied in a simple model [25]. Consider a shear flow with $x$ pointing in the direction of flow, $y$ in the normal direction with the shear and $z$ in the spanwise direction. Then the background velocity field with a linear shear is given by $\mathbf{U}_{0}=S y \mathbf{e}_{x}$. Adding as a perturbation the superposition of a vortex $\omega$ and a streak $\sigma$,

$$
\mathbf{u}=\omega(t)\left(\begin{array}{c}
0 \\
\beta \sin \alpha y \sin \beta z \\
\alpha \cos \alpha y \cos \beta z
\end{array}\right)+\sigma(t)\left(\begin{array}{c}
-\beta \sin \alpha y \sin \beta z \\
0 \\
0
\end{array}\right)
$$



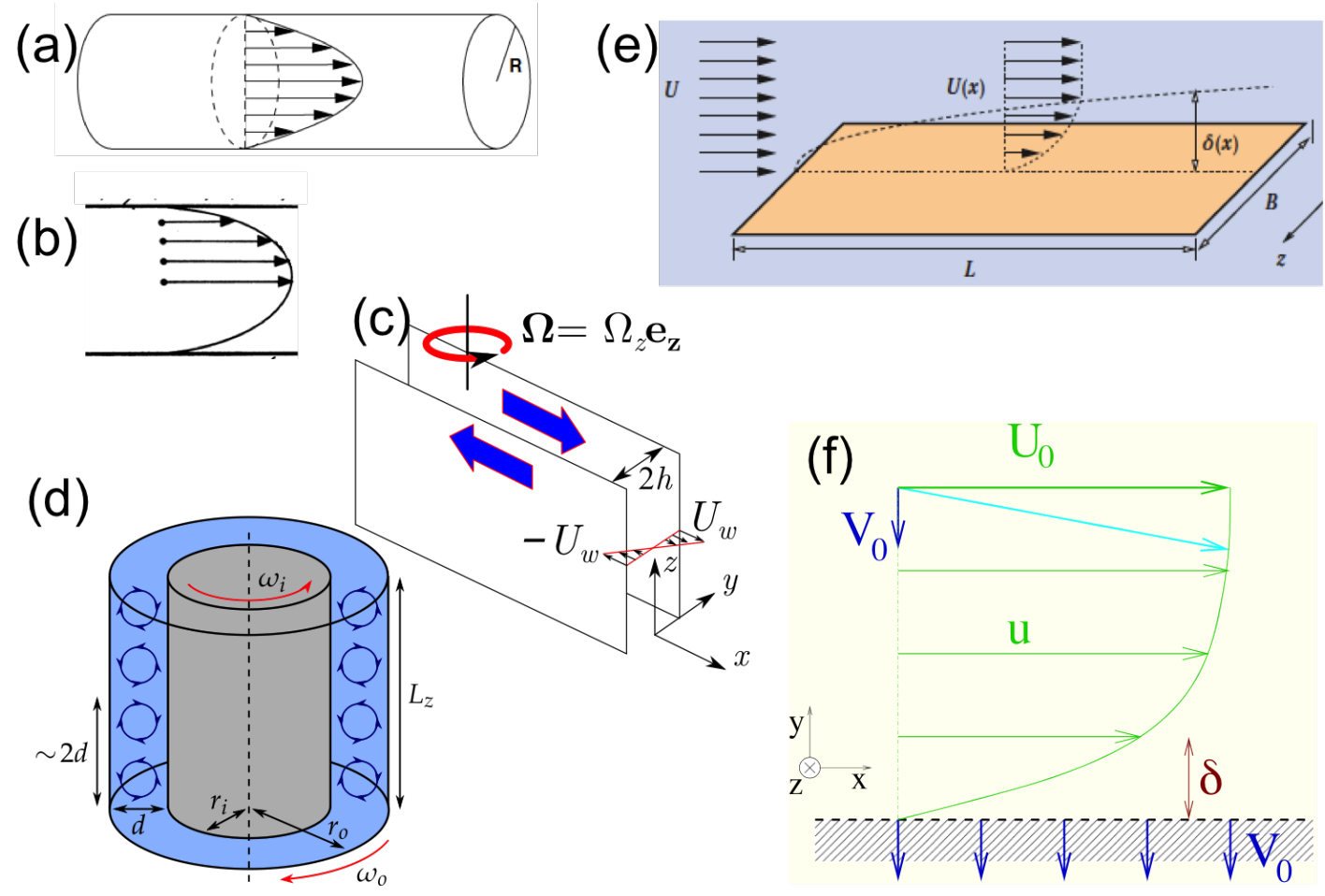

FIG. 1. (Colour online) Examples of flows where the transition to turbulence is induced by sufficiently strong perturbations and is not connected with a linear instability of the laminar profile. The left column shows internal flows bounded by walls on two sides, from top to bottom: (a) pressure driven flow in a cylindrical pipe of circular cross section (Hagen-Poiseuille flow); (b) the related pressure driven flow between parallel plates (plane Poiseuille flow); (c) shear flow between parallel walls moving relative to each other on a rotating table (rotating plane Couette flow); (d) flow between two independently rotating concentric cylinders (Taylor-Couette flow). The right column shows external flows bounded by a wall on one side only: (e) flow over a flat plate where the boundary layer increases with distance from the edge (Blasius) and (f) the suction boundary layer where a cross-flow maintains a parallel boundary layer of constant thickness. Of the flows shown here, plane Poiseuille flow, Taylor-Couette, and both boundary layer flows also have linear instabilites that become relevant in certain parameter ranges. The focus here is on transitions in parameter ranges without linear instabilites. Coordinates are usually chosen such that $x$ points in the flow direction, $y$ in the direction with the shear and $z$ in the spanwise neutral direction.

The resulting equations for the perturbation become

$$
\partial_{t}\left(\begin{array}{c}
\sigma \\
\omega
\end{array}\right)=\left(\begin{array}{cc}
-\Lambda & S \\
0 & -\Lambda
\end{array}\right)\left(\begin{array}{c}
\sigma \\
\omega
\end{array}\right)
$$

with the damping constant $\Lambda=\nu\left(\alpha^{2}+\beta^{2}\right)$. For the initial condition $(\sigma, \omega)(0)=(0,1)$ of a vortex but no streak, the time evolution becomes

$$
\left(\begin{array}{c}
\sigma(t) \\
\omega(t)
\end{array}\right)=\left(\begin{array}{c}
S t e^{-\Lambda t} \\
e^{-\Lambda t}
\end{array}\right)
$$

The linear increase in the streak is a consequence of the ability of the vortex to extract energy from the background shear flow. However, the flow will ultimately decay: all perturbations that are translationally invariant in the downstream direction will decay [26. The flow is therefore asympotically stable (in linear approximation).

More stringent than asymptotic stability is the requirement of energy stability, i.e. that the energy content in all perturbations has to decay monotonically, $d / d t \int\left(\mathbf{u}^{2} / 2\right) d V<0$, Ref. [27. For the above model, energy stability is possible for $S<2 \Lambda$. If the shear $S$ becomes sufficiently strong, the vortices are able to deposit enough energy in the streaks to given a transient increase in the the total energy. Detailed linear stability analyses of various shear flows and their transient amplification that take into account the correct boundary conditions are given in 28 31 .

A very interesting problem arises when the flows are studied in the presence of noise, 32 36. As shown for a simple model in Ref. [37, the noisy forcing of vortices can deposit more energy in the streaks than the noisy forcing, 
thus explaining the prominence of streaks in noisy shear flows. A dramatic consequence of this amplification has been studied by Luchini [38, 39]: The Navier-Stokes equations are continuum equations that average over microscopic molecular fluctuations. The microscopic constituents give rise to thermodynamic fluctuations around the continuum fields that are always present and increase with temperature [40. If there are processes that amplify the fluctuations by $O(\mathrm{Re})$, it is possible that thermodynamic fluctuations are amplified so much that they cross the threshold for the transition to turbulence. Indeed, Luchini's estimates in Ref. 38, 39] show that thermal fluctuations are big enough to cause transition in boundary layers for $\mathrm{Re} \approx 6 \cdot 10^{6}$. Similarly, Meseguer and Trefethen [4] noted that non-normality also causes a catastrophic loss of numerical accuracy that requires special precautions for linear stability analyses of pipe flow beyond $\mathrm{Re} \approx 10^{7}$. These values are only a little bit higher than the highest Reynolds number of order $O\left(10^{5}\right)$ up to which laminar pipe flow has been realized in experiments [42, 43.

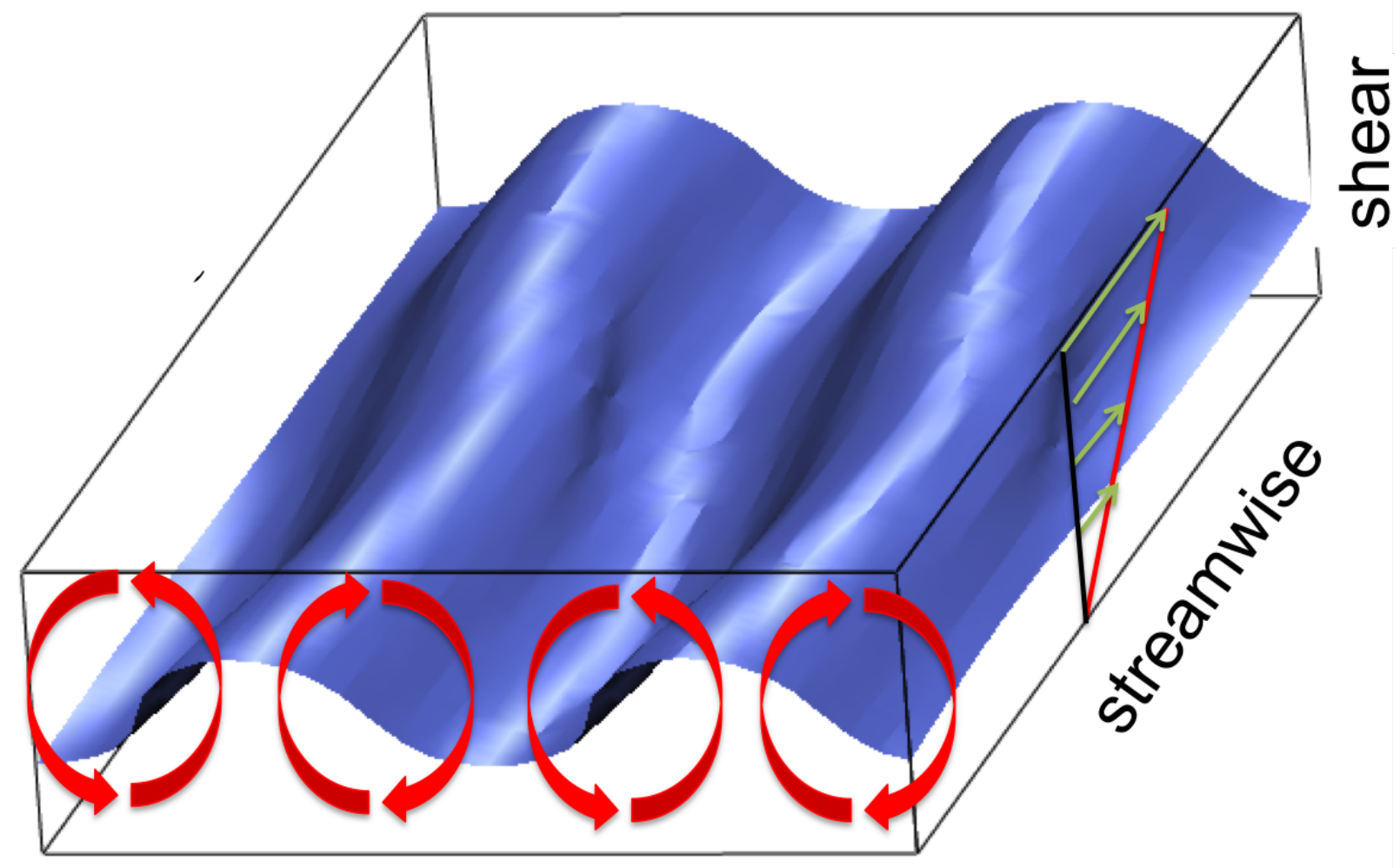

\section{spanwise}

FIG. 2. (Colour online) A sketch of the building blocks of turbulence in shear flows: vortices that are predominantly oriented in the streamwise direction mix the fluid across the shear and drive a streak, a modulation in the streamwise velocity. The blue surface shows the positions where the streamwise velocity equals the mid-plane value of the laminar shear flow. The modulations in height are due to the vortices and the spanwise modulations indicate that the structures are fully three-dimensional.

\section{NONLINEAR EFFECTS AND BIFURCATIONS}

The transient amplification of the streaks by the vortices by itself will not be sufficient to drive turbulence, as all structures that are translationally invariant in the downstream direction are bound to decay 26. However, the built up of streaks holds the seed for the formation of a linear instability near the inflection point in the downstream profile in the shearing region between the streaks [44. That instability will introduce spanwise modulations in the downstream direction, i.e. rather than being straight in the downstream direction, the vortices begin to show some modulation, and the modulated waves are capable of sustaining themselves. An example of the modulated exact coherent structures in plane Couette flow is shown in Fig. 2 .

On a technical level, the appearance of 3-d structures can be shown by embedding the flow into a family of flows where one parameter is the background flow (as measured by its Reynolds number) and the other parameter is a 
forcing that sustains the streaks (or the vortices, either way is fine). Once the instability has set in and the structures that break the downstream translational invariance have been identified, the forcing of the streaks is reduced and $3 \mathrm{~d}$ exact coherent structures for the initial field are obtained. This technique was initially used by Clever and Busse [45] for Rayleigh Benard convection, where they applied a temperature difference to drive convection rolls, and by Nagata in rotating plane Couette flow [46, where the second parameter in addition to shear was rotation. For pipe flow, Ref. 47] used an artificial body force mimicking vortices, and Ref. [4] used a body force derived from linear modes that were maximally amplified. In general, the path to exact coherent structures does not seem to depend very much on the details of the forcing as long as it drives vortices and streaks of reasonable wavelengths that are sufficiently strong to initiate the secondary instability to the formation of persistent structures. Another numerical approach to determine exact coherent states tracks trajectories in the boundary between laminar and turbulent flows that evolve to invariant states in the boundary, a technique known as edge tracking [49].

An example of the bifurcations that take place in the state space of the system is shown in Fig. 3 , for the case of plane Couette flow [50. Initially, below the formation of the structures, all initial conditions return to the laminar profile indicated by the blue circle. As one approaches the critical point for the onset of the structure, one initial condition is slowed down and ends up in the critical point, indicated in red. For higher Reynolds numbers, a saddle point (yellow) and a node (red) appear. Further away from the saddle point the region that is attracted to the node becomes very thin [51. The saddle point has stable and unstable directions, with the unstable directions either pointing towards the laminar profile or to the new node. In the 2-d example shown the saddle has one unstable direction, and the node has only stable directions. In higher-dimensions that may be more complicated, and the saddle-node bifurcation may happen in an unstable subspace. However, the saddle always has at least one more unstable direction than the node.

The region in state space that will be affected by this bifurcation has the shape of a droplet, as indicated in (d): the picture is obtained by mapping out in a 2 -d cross section of state space the fate of initial conditions in that plane (Ref. [50]): in the blue regions all initial conditions return to the laminar profile. In the red/brown area they are attracted to the nodal state. The tail of the droplet shaped region becomes very thin and reaches out to large amplitudes. As the Reynolds number increases, the bulk region becomes wider. The secondary bifurcations take place within the region and have little effect on its shape, until the crisis bifurcation that rips the closed attracting region open and turns the attractor into an open chaotic saddle.

(a)

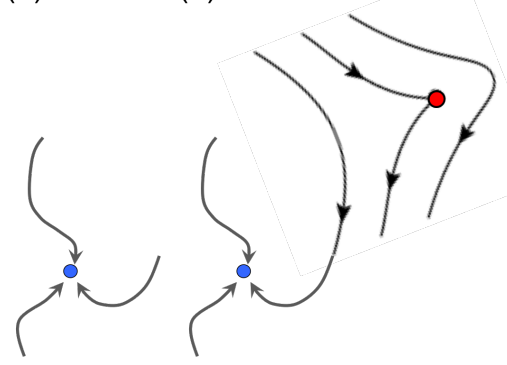

(c)

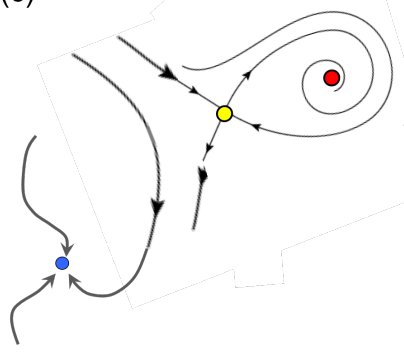

(d)

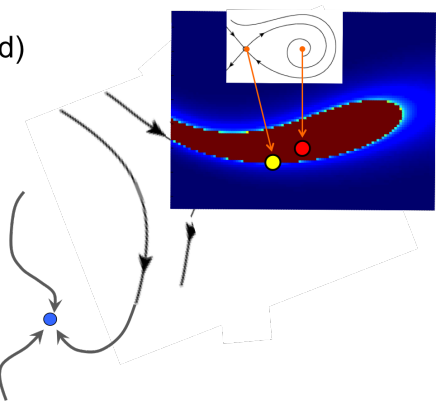

FIG. 3. (Colour online) A sketch of the saddle-node bifurcation in the state-space of the system. (a) Below the appearance of exact coherent structures, all initial conditions return to the laminar profile (blue dot), as indicated by the arrows. (b) At the critical point, one set of initial conditions converges to the new exact coherent structure (red dot). (c) Above the critical point a saddle (yellow) and a node (red) appear and a finite regions of initial conditions is attracted to the node. (d) The region attracted to the node can be mapped out by following the time evolution of initial conditions: in the blue region they return to the laminar profile, in the red region that approach the node. The figure is adapted from the results in [50].

The nodal state undergoes secondary bifurcations that add temporal complexity [50, 52, 53]: the bifurcation diagram shown in Fig. 4 looks very much like that of the logistic or quadratic map $x_{n+1}=4 \lambda x_{n}\left(1-x_{n}\right)$ as the parameter $\lambda$ is increased: a Hopf bifurcation brings in a frequency that turns the stationary states (in plane Couette flow) and the travelling waves (that are stationary states in a comoving frame of reference, in plane Poiseuille flow or pipe flow) into relative periodic orbits. Upon further increase in Reynolds number, they undergo a sequence of period doubling bifurcations, and then show a series of chaotic bands with periodic windows in between. Further increasing the Reynolds number results in a collision between the attractor that has formed around the node and a stable direction of the saddle. In such a crisis bifurcation [54 the attractor changes to a transient chaotic saddle. With the chaotic saddle comes the sensitive dependence on initial conditions seen in experiment [55] and simulations [56], as well as the possibility to decay and to return to the laminar flow [57, 58].

When additional parameters, such as the wavelength in the neutral directions, are taken into account, the unfolding 
of the saddle-node bifurcation becomes more complex [59, 60].

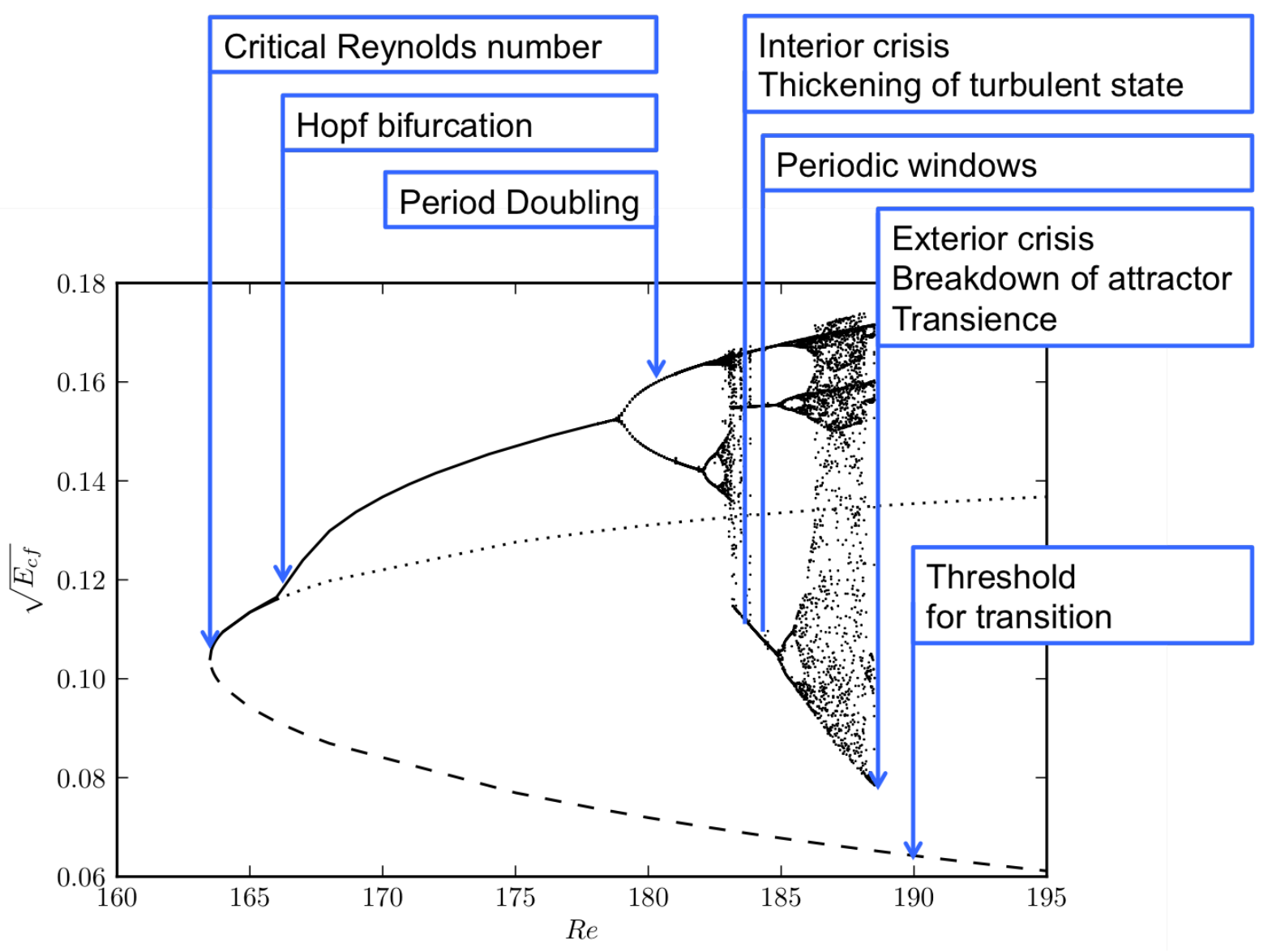

FIG. 4. (Colour online) Secondary bifurcations of exact coherent structures. The exact coherent structures appear at the critical Reynolds number, undergo a Hopf bifurcation followed by period doubling bifurcations, various interior crises in which the attractor becomes wider [61, the familiar periodic windows, and eventually a crisis bifurcation where the attractor collides with the saddle state (indicated by the dashed line) and turns into a strange saddle. The node also determines the threshold which perturbations have to cross in order to trigger turbulence.

The lack of stable coherent structures and the chaotic nature of the dynamics above onset make it very difficult to detect exact coherent structures in experiments. With judiciously chosen observables, it is possible to pick them out in velocity fields and to see the transitions between a few of these patterns 62 64. Moreover, it is possible extract velocity fields from observations and to continue their evolution numerically and to thereby verify that they stay close to invariant solutions for some time [65].

\section{LOCALIZATION}

The coexistence of a turbulent state (even if it is only transient) and a stable laminar state provides key ingredients for the formation of localized turbulent patches with transitions (fronts) between the laminar and turbulent state on their boundary. As an early example for shear flows, one finds the numerical experiment by Lundbladh and Johannsson [66]: they initiated a localized patch in plane Couette flow and studied its evolution in time for different Reynolds numbers. If the Reynolds number was too low, the patch would shrink and disappear. For higher Re, the patch would grow and expand in all directions. More extensive calculations by [12] showed that the patch does not stay uniform but develops alternating oblique bands of turbulent and laminar regions. Studies by Barkley and Tuckerman [67] obtained similar structures from homogeneous turbulent states upon decreasing the Reynolds number. Further examples of localized turbulence come from observations on puffs and slugs in pipe flow 68. (see Fig. 5) or spiral turbulence in Taylor-Couette flow [10]. 

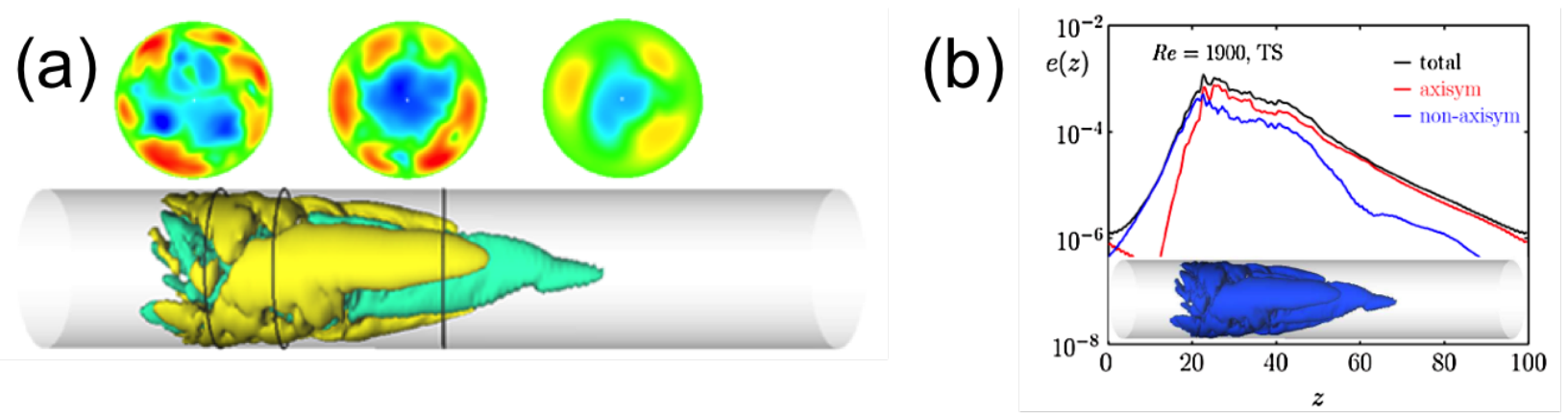

FIG. 5. (Colour online) A localized state in pipe flow, taken from [69]. (a) the highspeed streaks in the center (green) and the lowspeed streaks near the wall (yellow) together with cross sections of the pipe. (b) energy content of the puff along the axis of the pipe; the data are compatible with an exponential decay.

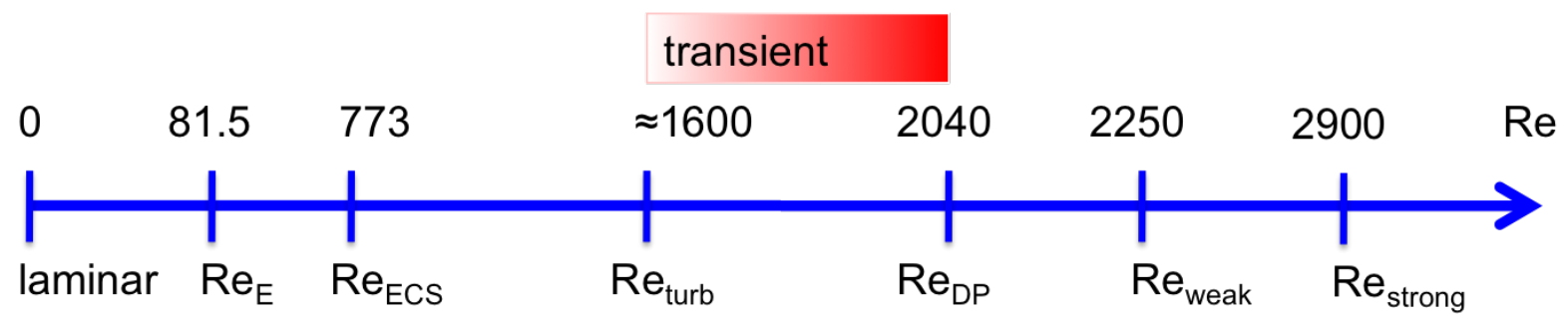

FIG. 6. (Colour online) The various Reynolds numbers of pipe flow along the Reynolds number axis in an updated version of the diagram from 80] (not to scale): energy stability $\operatorname{Re}_{E}=81.5$ [27]; appearance of the first critical states near Re $\mathrm{E}_{E S}=773$ (Ref. 81]); first indications of turbulence in experiments near $\approx 1600$ [18; critical Reynolds number from balance between splitting and decay $\operatorname{Re}_{D P} \approx 2040$ [82]; weak spreading of slugs above $\operatorname{Re} \approx 2250$; and finally transition to strong spreading near $\operatorname{Re}=2900$ 79.

The mechanisms by which a localized turbulent spot can spread differ in the spanwise and downstream direction. In the spanwise direction studies of spots between plates show that the longitudinal vortices at the boundary drive further rolls in their neighborhood [70 72. This process is not monotonic, and some of the rolls that are triggered may decay again, which is consistent with the transient nature of local structures. In the flow direction spreading is driven by the advection of turbulent blobs that are pulled out of the turbulent region.

The dynamics of localized spots is not restricted to such processes: it is also possible that the entire structure moves around. In the case of the suction boundary layer, various forms of localized, periodically oscillating or propagating and erratically meandering states have been found [73 75 . Similar structures have also been observed in plane Poiseuille flow 76, 77.

Pipe flow shows an even larger variety of structures: the first structures that appear, so-called puffs [78, do not grow to fill the pipe but maintain a certain length (the example shown in Fig. 5). For higher Reynolds numbers, they show a transition to another form that differs in the intensity profiles along the axis, so-called slugs. For slugs, the fronts move at different speeds so that they spread along the pipe. As more detailed theoretical and experimental studies show [21, 79], there are two kinds of slugs, distinguished by details of their intensity profiles and their spreading speeds. The characterization of weak and strong slugs follows from intriguing connections to the nonlinear dynamics of pulse propagation on nerve cells and raises interesting questions about frontal dynamics, as explained in Barkleys valuable perspectives article [21].

The relation between large scale coherent structures and the extended coherent structures described in the previous section is only partly understood. Studies on structures in large domains 83 87 show that extended structures are susceptible to long wavelength modulations which trigger transitions to localized structures. The connection to snaking bifurcations [88 90] suggests that there are entire families of localized structures with different numbers of vortices and different lengths and widths. In the downstream direction, the decay of the structures tends to be exponential, as shown in Refs. 91 94 using approximate representations of the velocity fields. The full analysis using representations for incompressible flow fields and allowing for modulations in the decay, is described in [95. In the spanwise direction, the decay seems to be even more rapid. In two-dimensional situations, e.g. with localized 
structures in plane Couette flow 72, one finds large scale flow structures that decay more slowly. The decay of states is not only of interest in its own right but also controls the interactions between patterns and therefore has implications for the relation to non-equilibrium phase transitions that described in the next section.

\section{SPATIOTEMPORAL DYNAMICS AND DIRECTED PERCOLATION}

The coexistence of the stable laminar state and a non-persistent turbulent state allows for spatio-temporal dynamics that is reminiscent of directed percolation, a paradigmatic non-equilibrium phase transition 96. Observations of puffs show that while they decay after some time [58, 97], they can also nucleate another puff when a turbulent blob is extracted, pulled downstream by the mean shear, and then reattached to the wall. If a puff is more likely to decay than to nucleate another one, the total number of puffs will decrease until the laminar profile is restored. If the puffs will split sufficiently frequently before they decay, then the number of puffs will increase and a finite fraction of space will remain turbulent. As described in [82, both processes can be viewed as independent random events, with exponential distributions in the times before an event (decay or split) occurs. Avila et al [82] found that the mean time $T_{d}$ for a decay increases with Reynolds number like

$$
T_{d} \approx \exp \exp \left(\frac{\operatorname{Re}-1550}{180}\right)
$$

and the mean time for a splitting event decays with Reynolds number like

$$
T_{s} \approx \exp \exp \left(\frac{2940-\mathrm{Re}}{320}\right)
$$

The stronger than exponential variation of life times with Reynolds number is robust and has also been seen in other systems. To date, the most plausible explanation for the super-exponential variation uses a link to large fluctuations and rare events [98, and such arguments could also motivate the faster than exponential variation of the time to a splitting event. Equating the decay and splitting times gives an estimate for a Reynolds number where the two processes balance: $\operatorname{Re}_{c} \approx 2040$. For $\operatorname{Re}$ below $\operatorname{Re}_{c}$ all turbulent patches will eventually decay and the system will return to the laminar profile. This definition of a critical Reynolds number can be used to replace the variety of critical Reynolds numbers that can be found in the literature [99] and it can also be used for other flows [100].

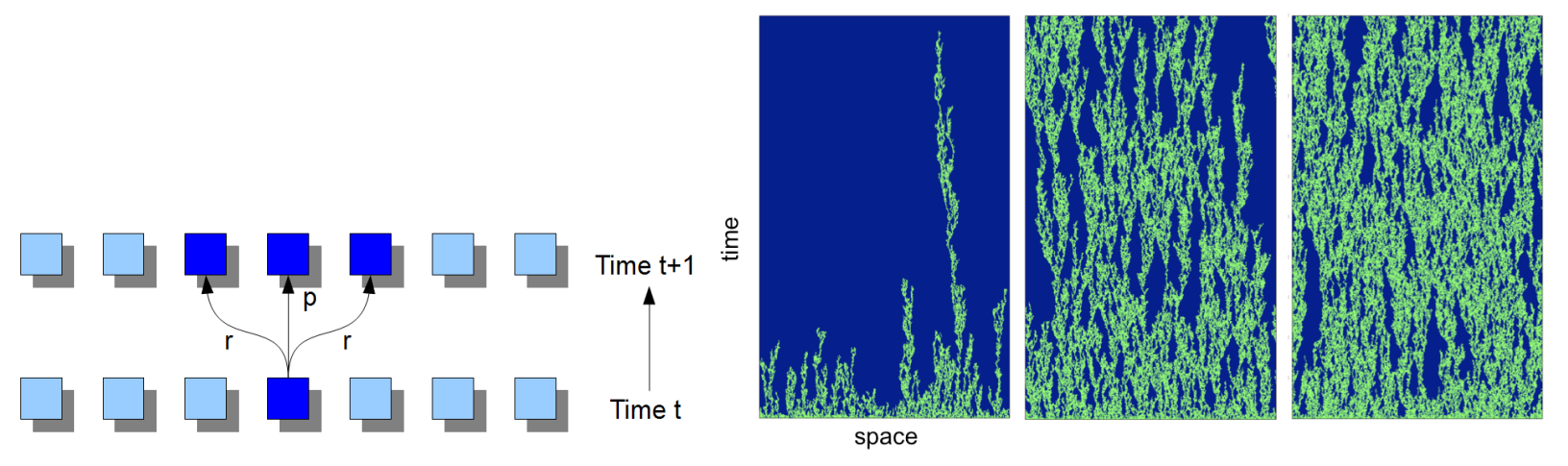

FIG. 7. (Colour online) The dynamics in 1d directed percolation. (a) a turbulent cell can stay turbulent (with probability $p$ ) and introduce turbulence in the neighboring cells (with probability $r$ ). (b) typical space-time diagrams in directed percolation following the dynamics in (a). The light green areas are turbulent cells. The first frame is below the critical point where all turbulent cells die out. The middle frame is at the critical point, and the right frame is above the critical point.

The dynamics of decay and splits in the turbulent puffs is reminiscent of the dynamics in directed percolation, thereby providing a first realization of a suggestion of Pomeau 101 103. Directed percolation is a time-discrete process on a discrete lattice: states are labelled laminar or turbulent, and a turbulent state can remain turbulent with a certain probability $p$ and induce transitions in neighboring cells to become turbulent with another probability $r$ (see Fig. 7 for the model studied in [104]. Identifying a puff with the turbulent state, the decay and spreading mimick the dynamics in directed percolation. The Reynolds number is the control parameter and the critical Reynolds number marks the transition point. The turbulent fraction $f$ can be taken as the order parameter for the transition, and it varies for 1-d percolation like

$$
f \propto\left(\operatorname{Re}-\operatorname{Re}_{c}\right)^{0.276}
$$


Other observables like the length and width of laminar regions scale with different exponents [96. The exponents have not been measured for pipe flow because of the excessively large observation times. However, the critical exponents could be measured in a thin Taylor-Couette cell [14, and all exponents have been found to be in good agreement with theoretical predictions.

In pipe flow, and also in duct flow or narrow Taylor-Couette flow, the turbulence is by and large homogeneous across a cross section, so that only one degree of freedom, along the axis, has to be taken into account. In the twodimensional cases like plane Couette flow or plane Poiseuille flow [13, the turbulent regions are not homogeneous, and there are observations that indicate that there several non-laminar states [105, 106]. Therefore, the relation to directed percolation in $2 \mathrm{~d}$ systems is less clear [102, 107].

\section{CONCLUSIONS AND OUTLOOK}

The past decades have seen much progress in our understanding of the transition to turbulence in shear flows without linear instabilities. The presence of shear drives vortices and streaks so that the energy content in the perturbations is transiently amplified. The amplification shows how shear flows become more susceptible to perturbations as the flow rate increases. Eventually, nonlinear effects become important and new, three-dimensional states appear in subcritical bifurcations. They provide a scaffold that can support turbulent dynamics: phrased the other way around, no turbulence is possible until such states are present. The coexistence of the new states with the stable laminar profile allows for the development of spatially localized patterns, such as the puffs and slugs in pipe flow.

Initially, the regions in state space that are affected by the new states are small, but as ever more states appear, they connect and entangle to form a web that can sustain turbulence for long times [90, 108, 109]. In spatially extended systems a variety of patterns can arise, with complex dynamics between different patterns and on the boundary to the laminar profile. The coexistence of the turbulent dynamics with a stable laminar profile suggests links to the non-equilibrium phase transition of directed percolation, and for $1 \mathrm{~d}$ systems there is evidence that the transition has all the characteristics of directed percolation. It remains a challenge to establish similar results for transition in $2 \mathrm{~d}$ flows.

One way to summarize the different processes and transitions is the sequence of Reynolds numbers shown in Fig. 6. It will be interesting to explore similar sequences and processes in other flows.

All the examples described here are parallel shear flows that are translationally invariant in the downstream direction. For spatially developing flows, such as boundary layers, the local variation in Reynolds numbers has to be taken into account. Some of the concepts described above can be transferred also to boundary layers [110, 111], and steps towards the explanation of the spatiotemporal fluctuations near the transition [112, 113] have been taken [114 117].

For higher Reynolds numbers beyond the transition to turbulence, the challenging question is the emergence of the turbulent cascade. For this, the dynamics has to become not only temporally but also spatially complex . It will be interesting to see to which extend the ideas developed for the transition can be carried over to the statistical mechanics of the turbulent cascade.

\section{ACKNOWLEDGEMENT}

This work has been supported in part by the Deutsche Forschungsgemeinschaft within its priority program "Turbulent superstructures".

[1] L. D. Landau, On the problem of turbulence, Dokl Akad Nauk SSSR 44 (1944) 311.

[2] F. H. Busse, Non-linear properties of thermal convection, Rep Prog Phys 41 (1978) 1929-1967.

[3] E. L. Koschmieder, Bénard Cells and Taylor Vortices, Cambridge University Press, 1993.

[4] M. C. Cross, Hohenberg, P. C., Pattern formation outside of equilibrium, Rev Mod Phys 65 (3) (1993) 851-1112.

[5] I. S. Aranson, L. Kramer, The world of the complex Ginzburg-Landau equation, Rev Mod Phys 74 (1) (2002) 99.

[6] P. Manneville, Dissipative Structures and Weak Turbulence, Academic Press, 2014.

[7] O. Reynolds, An experimental investigation of the circumstances which determine whether the motion of water shall be direct or sinuous, and of the law of resistance in parallel channels., Proc R Soc (London) 35 (1883) 84-99.

[8] O. Reynolds, An Experimental Investigation of the Circumstances which Determine Whether the Motion of Water Shall be Direct Or Sinuous, Phil Trans R Soc (London) 174 (1883) 935-982.

[9] S. Grossmann, The onset of shear flow turbulence, Rev Mod Phys 72 (2000) 603-618. 
[10] A. Prigent, G. Grégoire, H. Chaté, O. Dauchot, W. van Saarloos, Large-Scale Finite-Wavelength Modulation within Turbulent Shear Flows, Phys Rev Lett 89 (2002) 014501.

[11] D. Moxey, D. Barkley, Distinct large-scale turbulent-laminar states in transitional pipe flow, Proc Nat Acad Sci USA 107 (2010) 8091-8096.

[12] Y. Duguet, P. Schlatter, D. S. Henningson, Formation of turbulent patterns near the onset of transition in plane Couette flow, J Fluid Mech 650 (2010) 119-129.

[13] M. Sano, K. Tamai, A universal transition to turbulence in channel flow, Nat Phys 12 (2016) $249-253$.

[14] G. Lemoult, L. Shi, K. Avila, S. V. Jalikop, M. Avila, B. Hof, Directed percolation phase transition to sustained turbulence in Couette flow, Nat Phys 12 (2016) 254-258.

[15] R. R. Kerswell, Recent progress in understanding the transition to turbulence in a pipe, Nonlinearity 18 (2005) R17-R44.

[16] B. Eckhardt, Turbulence transition in pipe flow: some open questions, Nonlinearity 21 (2007) T1-T11.

[17] B. Eckhardt, T. M. Schneider, B. Hof, J. Westerweel, Turbulence Transition in Pipe Flow, Annu Rev Fluid Mech 39 (2007) 447-468.

[18] A. P. Willis, J. Peixinho, R. R. Kerswell, T. Mullin, Experimental and theoretical progress in pipe flow transition, Phil Trans R Soc A 366 (2008) 2671-2684.

[19] T. Mullin, Experimental Studies of Transition to Turbulence in a Pipe, Annu Rev Fluid Mech 43 (1) (2011) 1-24.

[20] G. Kawahara, M. Uhlmann, L. van Veen, The Significance of Simple Invariant Solutions in Turbulent Flows, Annu Rev Fluid Mech 44 (2012) 203-225.

[21] D. Barkley, Theoretical perspective on the route to turbulence in a pipe, J Fluid Mech 803 (2016) P1.

[22] P. Manneville, Laminar-Turbulent Patterning in Transitional Flows, Entropy 19 (2017) 316.

[23] J. F. Gibson, J. Halcrow, P. Cvitanović, Equilibrium and travelling-wave solutions of plane Couette flow, J Fluid Mech 638 (2009) 243-266.

[24] B. Song, B. Hof, Deterministic and stochastic aspects of the transition to turbulence, J Stat Mech: Theory Exp 2014 (2014) P02001.

[25] B. Eckhardt, R. Pandit, Noise correlations in shear flows, Eur Phys J B 33 (2003) 373-378.

[26] H. K. Moffatt, Fixed points of turbulent dynamical systems and suppression of nonlinearity Comment 1, in: Whither Turbulence? Turbulence at the Crossroads: Proceedings of a Workshop Held at Cornell University, Department of Applied Mathematics and Theoretical Physics, 1990, pp. 250-257.

[27] D. D. Joseph, S. Carmi, Stability of Poiseuille flow in pipes, annuli, and channels, Quart Appl Math 26 (1969) 575-599.

[28] P. J. Schmid, D. S. Henningson, Stability and Transition of Shear Flows, Springer, New York, 1999.

[29] L. Boberg, U. Brosa, Onset of turbulence in a pipe, Z Naturf A 43 (1988) 697-726.

[30] L. N. Trefethen, A. E. Trefethen, S. C. Reddy, T. A. Driscoll, Hydrodynamic stability without eigenvalues, Science 261 (1993) 578-584.

[31] S. C. Reddy, P. J. Schmid, D. S. Henningson, Pseudospectra of the Orr-Sommerfeld Operator, SIAM J Appl Math 53 (1993) 15-47.

[32] J. M. Ortiz de Zárate, J. V. Sengers, Transverse-velocity fluctuations in a liquid under steady shear, Phys Rev E 77 (2008) 026306 .

[33] J. M. Ortiz de Zárate, J. Sengers, Nonequilibrium velocity fluctuations and energy amplification in planar Couette flow, Phys Rev E 79 (2009) 046308.

[34] J. V. Sengers, J. M. Ortiz de Zárate, Velocity fluctuations in laminar fluid flow, J Non-Newt Fluid Mech 165 (2010) 925-931.

[35] J. M. Ortiz de Zárate, J. V. Sengers, Hydrodynamic Fluctuations in Laminar Fluid Flow. I. Fluctuating Orr-Sommerfeld Equation, J Stat Phys 144 (2011) 774-792.

[36] J. M. Ortiz de Zárate, J. V. Sengers, Hydrodynamic Fluctuations in Laminar Fluid Flow. II. Fluctuating Squire Equation, J Stat Phys 150 (2012) 540-558.

[37] M. Pausch, B. Eckhardt, Theoretical and Applied Mech Letters, Theor. Appl. Mech. Lett. 5 (2015) $111-116$.

[38] P. Luchini, A Thermodynamic Lower Bound on Transition-Triggering Disturbances, Seventh IUTAM Symposium on Laminar-Turbulent Transition, Springer 2010, pg. 11-18.

[39] P. Luchini, Receptivity to Thermal Noise of the Boundary Layer over a Swept Wing, AIAA Journal 55 (2017) 121-130.

[40] L. D. Landau, E. M. Lifshitz, Statistical Physics: V. 5: Course of Theoretical Physics, Pergamon press, 1969.

[41] A. Meseguer, L. N. Trefethen, Linearized pipe flow to Reynolds number 107, J Comp Phys 186 (2003) $178-197$.

[42] W. Pfenniger, Transition in the inlet length of tubes at high Reynolds numbers, Boundary layer and flow control (ed. GV Lachman) (1961) 970-980.

[43] A. A. Draad, G. Kuiken, F. T. M. Nieuwstadt, Laminar-turbulent transition in pipe flow for Newtonian and non-Newtonian fluids, J Fluid Mech 377 (1998) 267-312.

[44] F. Waleffe, On a self-sustaining process in shear flows, Phys Fluids A 9 (1997) 883-900.

[45] R. M. Clever, F. H. Busse, Tertiary and quaternary solutions for plane Couette flow, J Fluid Mech 344 (1997) 137-153.

[46] M. Nagata, Three-dimensional finite-amplitude solutions in plane Couette flow : bifurcation from infinity, J Fluid Mech 217 (1990) 519-527.

[47] H. Faisst, B. Eckhardt, Traveling Waves in Pipe Flow, Phys Rev Lett 91 (2003) 224502.

[48] H. Wedin, R. R. Kerswell, Exact coherent structures in pipe flow: travelling wave solutions, J Fluid Mech 508 (2004) 333-371.

[49] J. D. Skufca, J. A. Yorke, B. Eckhardt, Edge of Chaos in a Parallel Shear Flow, Phys Rev Lett 96 (2006) 174101.

[50] T. Kreilos, B. Eckhardt, Periodic orbits near onset of chaos in plane Couette flow, Chaos 22 (2012) 047505. 
[51] N. R. Lebovitz, Shear-flow transition: the basin boundary, Nonlinearity 22 (2009) 2645-2655.

[52] S. Zammert, B. Eckhardt, Crisis bifurcations in plane Poiseuille flow, Phys Rev E 91 (2015) 041003.

[53] M. Avila, F. Mellibovsky, N. Roland, B. Hof, Streamwise-Localized Solutions at the Onset of Turbulence in Pipe Flow, Phys Rev Lett 110 (2013) 224502.

[54] C. Grebogi, E. Ott, J. A. Yorke, Chaotic Attractors in Crisis, Phys Rev Lett 48 (1982) 1507-1510.

[55] A. G. Darbyshire, T. Mullin, Transition to turbulence in constant-mass-flux pipe flow, J Fluid Mech 289 (1995) 83-114.

[56] A. Schmiegel, B. Eckhardt, Fractal stability border in plane Couette flow, Phys Rev Lett 79 (1997) 5250.

[57] H. Faisst, B. Eckhardt, Sensitive dependence on initial conditions in transition to turbulence in pipe flow, J Fluid Mech 504 (2004) 343-352.

[58] B. Hof, J. Westerweel, T. M. Schneider, B. Eckhardt, Finite lifetime of turbulence in shear flows., Nature 443 (2006) $59-62$.

[59] F. Mellibovsky, B. Eckhardt, Takens-Bogdanov bifurcation of travelling-wave solutions in pipe flow, J Fluid Mech 670 (2011) 96-129.

[60] F. Mellibovsky, B. Eckhardt, From travelling waves to mild chaos: a supercritical bifurcation cascade in pipe flow, J Fluid Mech 709 (2012) 149-190.

[61] T. Kreilos, B. Eckhardt, T. M. Schneider, Increasing Lifetimes and the Growing Saddles of Shear Flow Turbulence, Phys Rev Lett 112 (2014) 044503.

[62] B. Hof, C. W. H. van Doorne, J. Westerweel, F. T. M. Nieuwstadt, H. Faisst, B. Eckhardt, H. Wedin, R. R. Kerswell, F. Waleffe, Experimental Observation of Nonlinear Traveling Waves in Turbulent Pipe Flow, Science 305 (2004) 15941598 .

[63] T. M. Schneider, B. Eckhardt, J. Vollmer, Statistical analysis of coherent structures in transitional pipe flow, Phys Rev E 75 (2007) 066313.

[64] R. R. Kerswell, O. R. Tutty, Recurrence of travelling waves in transitional pipe flow, J Fluid Mech 584 (2007) 69-102.

[65] A. De Lozar, F. Mellibovsky, M. Avila, B. Hof, Edge state in pipe flow experiments, Phys Rev Lett 108 (2012) 214502.

[66] A. Lundbladh, A. V. Johansson, Direct simulation of turbulent spots in plane Couette flow, J Fluid Mech 229 (1991) 499-516.

[67] D. Barkley, L. S. Tuckerman, Computational Study of Turbulent Laminar Patterns in Couette Flow, Phys Rev Lett 94 (2005) 014502.

[68] J. Rotta, Experimenteller Beitrag zur Entstehung turbulenter Strömungen im Rohr, Ingenieur-Archiv 24 (1956) $258-281$.

[69] F. Mellibovsky, A. Meseguer, T. M. Schneider, B. Eckhardt, Transition in Localized Pipe Flow Turbulence, Phys Rev Lett 103 (2009) 054502.

[70] Y. Duguet, O. Le Maître, P. Schlatter, Stochastic and deterministic motion of a laminar-turbulent front in a spanwisely extended Couette flow, Phys Rev E 84 (2011) 066315.

[71] Y. Duguet, P. Schlatter, Oblique laminar-turbulent interfaces in plane shear flows, Phys Rev Lett 110 (2013) 034502.

[72] J. Schumacher, B. Eckhardt, Evolution of turbulent spots in a parallel shear flow, Phys Rev E 63 (2001) 046307.

[73] T. Khapko, T. Kreilos, P. Schlatter, Y. Duguet, B. Eckhardt, D. S. Henningson, Localized edge states in the asymptotic suction boundary layer, J Fluid Mech 717 (2013) R6.

[74] T. Khapko, Y. Duguet, T. Kreilos, P. Schlatter, B. Eckhardt, D. S. Henningson, Complexity of localised coherent structures in a boundary-layer flow, Eur Phys J E 37 (2014) 1-12.

[75] T. Khapko, T. Kreilos, P. Schlatter, Y. Duguet, B. Eckhardt, D. S. Henningson, Edge states as mediators of bypass transition in boundary-layer flows, J Fluid Mech 801 (2016) R2.

[76] L. S. Tuckerman, T. Kreilos, H. Schrobsdorff, T. M. Schneider, J. F. Gibson, Turbulent-laminar patterns in plane Poiseuille flow, Phys Fluids A 26 (2014) 114103.

[77] X. Xiong, J. Tao, S. Chen, L. Brandt, Turbulent bands in plane-Poiseuille flow at moderate Reynolds numbers, Phys Fluids A 27 (2015) 041702.

[78] I. J. Wygnanski, F. H. Champagne, On transition in a pipe. Part 1. The origin of puffs and slugs and the flow in a turbulent slug, J Fluid Mech 59 (1973) 281-335.

[79] D. Barkley, B. Song, V. Mukund, G. Lemoult, M. Avila, B. Hof, The rise of fully turbulent flow, Nature 526 (2015) $550-553$.

[80] B. Eckhardt, A Critical Point for Turbulence, Science 333 (2011) 165-166.

[81] C. C. T. Pringle, R. R. Kerswell, Asymmetric, Helical, and Mirror-Symmetric Traveling Waves in Pipe Flow, Phys Rev Lett 99 (2007) 074502.

[82] K. Avila, D. Moxey, A. de Lozar, M. Avila, D. Barkley, B. Hof, The Onset of Turbulence in Pipe Flow, Science 333 (2011) 192-196.

[83] Y. Duguet, P. Schlatter, D. S. Henningson, Localized edge states in plane Couette flow, Phys Fluids A 21 (2009) 111701.

[84] T. M. Schneider, D. Marinc, B. Eckhardt, Localized edge states nucleate turbulence in extended plane Couette cells, J Fluid Mech 646 (2010) 441.

[85] K. Melnikov, T. Kreilos, B. Eckhardt, Long-wavelength instability of coherent structures in plane Couette flow, Phys Rev E 89 (2014) 043008.

[86] M. Chantry, A. P. Willis, R. R. Kerswell, Genesis of Streamwise-Localized Solutions from Globally Periodic Traveling Waves in Pipe Flow, Phys Rev Lett 112 (2014) 164501.

[87] F. Mellibovsky, A. Meseguer, A mechanism for streamwise localisation of nonlinear waves in shear flows, J Fluid Mech 779 (2015) R1.

[88] E. Knobloch, Spatially localized structures in dissipative systems: open problems, Nonlinearity 21 (2008) T45-T60. 
[89] T. M. Schneider, J. F. Gibson, J. Burke, Snakes and Ladders: Localized Solutions of Plane Couette Flow, Phys Rev Lett 104 (2010) 104501.

[90] J. F. Gibson, T. M. Schneider, Homoclinic snaking in plane Couette flow: bending, skewing and finite-size effects, J Fluid Mech 794 (2016) 530-551.

[91] J. F. Gibson, E. Brand, Spanwise-localized solutions of planar shear flows, J Fluid Mech 745 (2014) 25-61.

[92] E. Brand, J. F. Gibson, A doubly localized equilibrium solution of plane Couette flow, J Fluid Mech 750 (2014) R3.

[93] S. Zammert, B. Eckhardt, Streamwise decay of localized states in channel flow, Phys Rev E 94 (2016) 041101(R).

[94] P. Ritter, S. Zammert, B. Song, B. Eckhardt, M. Avila, Analysis and modeling of localized invariant solutions in pipe flow, Phys Rev Fluids 3 (2018) 1-17.

[95] J. Barnett, D. R. Gurevich, R. O. Grigoriev, Streamwise localization of traveling wave solutions in channel flow, Phys Rev E 95 (2017) 033124.

[96] H. Hinrichsen, Non-equilibrium critical phenomena and phase transitions into absorbing states, Adv Phys 49 (2000) $815-958$.

[97] B. Hof, A. de Lozar, D. Kuik, J. Westerweel, Repeller or Attractor? Selecting the Dynamical Model for the Onset of Turbulence in Pipe Flow, Phys Rev Lett 101 (2008) 214501.

[98] N. Goldenfeld, N. Guttenberg, G. Gioia, Extreme fluctuations and the finite lifetime of the turbulent state, Phys Rev E 81 (2010) 035304.

[99] B. Eckhardt, Introduction. Turbulence transition in pipe flow: 125th anniversary of the publication of Reynolds' paper, Phil Trans R Soc A 367 (2009) 449-455.

[100] L. Shi, M. Avila, B. Hof, Scale Invariance at the Onset of Turbulence in Couette Flow, Phys Rev Lett 110 (2013) 204502.

[101] Y. Pomeau, Front motion, metastability and subcritical bifurcations in hydrodynamics, Physica D 23 (1986) 3-11.

[102] Y. Pomeau, The long and winding road, Nat Phys 12 (2016) 198-199.

[103] P. Manneville, Spatiotemporal perspective on the decay of turbulence in wall-bounded flows, Phys Rev E 79 (2009) 025301.

[104] K. T. Allhoff, B. Eckhardt, Directed percolation model for turbulence transition in shear flows, Fluid Dyn Res 44 (2012) 031201.

[105] X. Xiong, J. Tao, S. Chen, L. Brandt, Turbulent bands in plane-poiseuille flow at moderate reynolds numbers, Phys Fluids 27 (2015) 041702.

[106] J. Tao, B. Eckhardt, X. Xiong, Extended localized structures and the onset of turbulence in channel flow, arXiv preprint arXiv:1709.07165, Phys Rev Fluids, (2018) in press.

[107] M. Chantry, L. S. Tuckerman, D. Barkley, Turbulent-laminar patterns in shear flows without walls, J Fluid Mech 791 (2016) R8.

[108] J. Halcrow, J. F. Gibson, P. Cvitanović, D. Viswanath, Heteroclinic connections in plane Couette flow, J Fluid Mech 621 (2009) 365-376.

[109] P. Ritter, F. Mellibovsky, M. Avila, Emergence of spatio-temporal dynamics from exact coherent solutions in pipe flow, New J Phys 18 (2016) 083031.

[110] S. Cherubini, P. De Palma, J. C. Robinet, A. Bottaro, Edge states in a boundary layer, Phys Fluids A 23 (2011) 051705.

[111] Y. Duguet, P. Schlatter, D. S. Henningson, B. Eckhardt, Self-Sustained Localized Structures in a Boundary-Layer Flow, Phys Rev Lett 108 (2012) 044501.

[112] H. W. Emmons, The laminar-turbulent transition in a boundary layer-Part I, J Aeronaut Sci 18 (1958) $490-498$.

[113] R. Narasimha, The laminar-turbulent transition zone in the boundary layer, Prog Aerospace Sci 22 (1985) 29-80.

[114] S. Cherubini, J. C. Robinet, A. Bottaro, P. De Palma, Optimal wave packets in a boundary layer and initial phases of a turbulent spot, J Fluid Mech 656 (2010) 231-259.

[115] N. Vinod, R. Govindarajan, Pattern of Breakdown of Laminar Flow into Turbulent Spots, Phys Rev Lett 93 (2004) 114501.

[116] N. Vinod, R. Govindarajan, The signature of laminar instabilities in the zone of transition to turbulence, J Turb 8 (2007) N2.

[117] T. Kreilos, T. Khapko, P. Schlatter, Y. Duguet, D. S. Henningson, B. Eckhardt, Bypass transition and spot nucleation in boundary layers, Phys Rev Fluids 1 (2016) 043602. 